\title{
VARIACIÓN DE LA ABUNDANCIA Y DIVERSIDAD DE AVES EN EL HUMEDAL LUCRE-HUACARPAY, QUISPICANCHI / CUSCO / PERÚ, DURANTE EL PERIODO DE “EL NIÑO" 2015 - 2016
}

\author{
VARIATION OF ABUNDANCE AND DIVERSITY OF BIRDS IN LUCRE- \\ HUACARPAY WETLAND, QUISPICANCHI / CUSCO / PERU, DURING THE \\ PERIOD OF "EL NIÑO" 2015 - 2016
}

\author{
Wilian N. Cárdenas ${ }^{1}$ y Liz B. Hurtado ${ }^{2}$
}

\begin{abstract}
Resumen
Desde el mes de septiembre de 2015 hasta agosto de 2016 se realizó un seguimiento mensual en las rutas de los seis espejos de agua que conforman el Humedal Lucre-Huacarpay (Quispicanchi / Cusco / Perú), con el objetivo de analizar la variación espacial y temporal de la abundancia y la diversidad de aves. Habiéndose determinado 84 especies, distribuidas en 14 órdenes, 29 familias y 67 géneros. Por el grado de residencia, 63 especies fueron residentes, 4 fueron temporales, 5 fueron ocasionales y 12 fueron migratorias. El espejo de agua con mayor riqueza de especies y valores más altos de los índices de diversidad alfa fue Waskar. Los meses con mayor número de especies fueron septiembre de 2015 y febrero de 2016 con 57 especies cada uno. El estimador de Chao-2 indica que se registró el $84.2 \%$ del total de especies posibles a ocurrir en el área durante el periodo de la evaluación. El análisis de varianza de la diversidad de Shannon-Wiener entre los espejos de agua y la prueba de Tukey-b indicaron que Unkapampa fue el que menos diversidad posee y difiere de los demás espejos de agua evaluados. La prueba T-Student demostró que no existe diferencia de la diversidad, abundancia ni riqueza de especies entre la época de secas y lluvias. Las 5 especies con mayor abundancia fueron Zonotrichia capensis (10.33\%), Chroicocephalus serranus (9.46\%), Fulica ardesiaca (8.22\%), Catamenia analis (6.27\%) y Gallinula galeata $(5.91 \%)$.
\end{abstract}

Palabras clave: humedal mesoandino, Lucre-Huacarpay, avifauna, comunidades, variación temporal.

\begin{abstract}
From September 2015 to August 2016, a monthly evaluation was carried out in the six lakes that are part of Lucre Huacarpay wetland (Quispicanchi / Cusco / Perú) aiming to analyze temporal and spatial variation in the abundance and diversity of birds. For this period, 84 species of birds have been registered,included in 14 orders, 29 families, and 67 genres. Regarding the degree of residence, 63 were residents, 4 were temporary, 5 were occasional and 12 were migratory. The lake with the highest species richness and highest values of alfa index diversity was Waskar. The months with the highest species richness were September 2015 and February 2016, with 57 species in each one. The Chao-2 estimator indicates that $84.2 \%$ of the total species likely to occur in the area during the evaluation period was registered. The analysis of variance of the Shannon-Wiener diversity index and Tukey-b test among the lakes indicate that Unkapampa has the lowest alfa diversity and differs from the others. The five species with the highest abundance were Zonotrichia capensis (10.34\%), Chroicocephalus serranus (9.47\%), Fulica ardesiaca (8.23\%), Catamenia analis $(6.27 \%)$ and Gallinula galeata $(5.92 \%)$.
\end{abstract}

Key words: andean wetlands, Lucre-Huacarpay, birds, communities, temporal variation.

\section{Introducción}

Los humedales se encuentran entre los ecosistemas más productivos del planeta (Cronk \& Fennessy, 2016), cumpliendo al mismo tiempo funciones ecológicas fundamentales para el hombre a través de los servicios ecosistémicos que ofrecen (World Resources Institute, 2005). También, son hábitat de importancia para muchas especies de aves que los utilizan como áreas para refugio, forrajeo y como sitios de reproducción, anidación y crianza (Stewart, 1996). Son ecosistemas determinantes para la supervivencia de aves migratorias (Bildstein et al., 1991; Gauthier et al., 2005) por ser sitios de descanso que les proveen de alimento y energía para realizar sus vuelos (Lyons \& Haig, 1997; Blanco, 1999; Perrins, 2011). 
La conservación y uso sostenible de los humedales y sus recursos es promovida por la Convención sobre los Humedales de Importancia Internacional, Convención de Ramsar (Ramsar, 1971). Uno de los sitios Ramsar en el Perú es el humedal LucreHuacarpay, que fue designado en el año 2006 debido a su importancia como hábitat para aves acuáticas. Siguiendo a Venero (2015), en el humedal LucreHuacarpay se han registrado 140 especies de aves, de ellas: 70 residentes, 23 migratorias, 24 ocasionales y 18 temporales, con dos especies endémicas del sur del Perú: Asthenes ottonis y Oreonympha nobilis. Sin embargo, no se tiene información acerca de la variación espacial y temporal de la avifauna en este humedal.

La integración de nuevas especies producto de la estacionalidad, afecta la diversidad de especies de aves en los humedales (Iannacone et al., 2010), estos cambios temporales están bien documentados en las costas del Pacífico, pero en ecosistemas altoandinos la información es escasa (Pulido \& Dourojeanni, 1990; Giraldo et al., 2002; Torres et al., 2006; Pulido, 2018). En la región altoandina, se da dos estaciones al año una de lluvias y otra de secas. La época de lluvias corresponde a los meses de septiembre a abril y coincide con la llegada de las aves migratorias a los humedales, tanto boreales como australes y la época de secas corresponde a los meses de mayo a agosto (Venero, 2015).

No obstante, esta estacionalidad puede verse afectada por fenómenos climáticos como El Niño, en particular la precipitación y disponibilidad de agua se ven dramáticamente reducidas en los Andes Tropicales, lo que impacta en la agricultura, producción de energía, suministro de agua para las poblaciones humanas y la funcionalidad de los ecosistemas (Posada-Marín et al., 2019). El efecto de El Niño en las poblaciones de aves ha sido estudiado principalmente en aves marinas y guaneras (Tovar \& García, 1982; Apaza \& Figari, 1999) y se desconoce su influencia en humedales andinos.

En el transcurso del año 2015 el comité multisectorial encargado del Estudio Nacional del Fenómeno El Niño - ENFEN, así como otras fuentes (Suarez-Ognio et al., 2015), revelaron la posibilidad de un nuevo evento de El Niño para el periodo 2015 2016, cuya ocurrencia fue confirmada posteriormente y caracterizada como el evento más caluroso del siglo (Jiménez-Muñoz et al., 2016). Por tanto, el objetivo de este trabajo es cuantificar y comparar la diversidad alfa $\mathrm{y}$ beta entre las rutas de avistamiento de aves en los seis espejos de agua y conocer los cambios temporales en la diversidad y abundancia de aves en el Humedal LucreHuacarpay de septiembre del 2015 a agosto del 2016, que coincide con el fenómeno de El Niño del mismo año.

\section{Materiales y métodos}

\section{Área de estudio}

El sitio Ramsar Humedal Lucre-Huacarpay se encuentra ubicado en el distrito de Lucre, provincia de Quispicanchi, departamento del Cusco, Perú. Geográficamente se ubica entre las coordenadas $13^{\circ} 35^{\prime} 56.17^{\prime \prime S} ; \quad 71^{\circ} 42^{\prime} 27.58^{\prime \prime} \mathrm{W}$ y $13^{\circ} 40^{\prime} 14.46^{\prime \prime} \mathrm{S}$; $71^{\circ} 45^{\prime} 16.80^{\prime \prime} \mathrm{W}$. Tiene una extensión de 1979.25 ha, con una altitud comprendida entre 4112 y $3075 \mathrm{msnm}$ y una precipitación total anual de $560 \mathrm{~mm} \mathrm{y}$ temperatura media anual de $13.4{ }^{\circ} \mathrm{C}$ (WorldClim Global Climate Data, 2016). De acuerdo con Venero (2015) el clima es templado y moderadamente frío, con temperaturas que varían de $1.5{ }^{\circ} \mathrm{C}$ a $22.8{ }^{\circ} \mathrm{C}$, descendiendo bajo cero en los meses de junio y agosto. El Humedal Lucre-Huacarpay está constituido por seis espejos de agua que son permanentes durante el año y en los que se realizaron las evaluaciones: Watón, Lucre, Huacarpay, Choquepuquio, Wascar y Unkapampa (Figura 1).

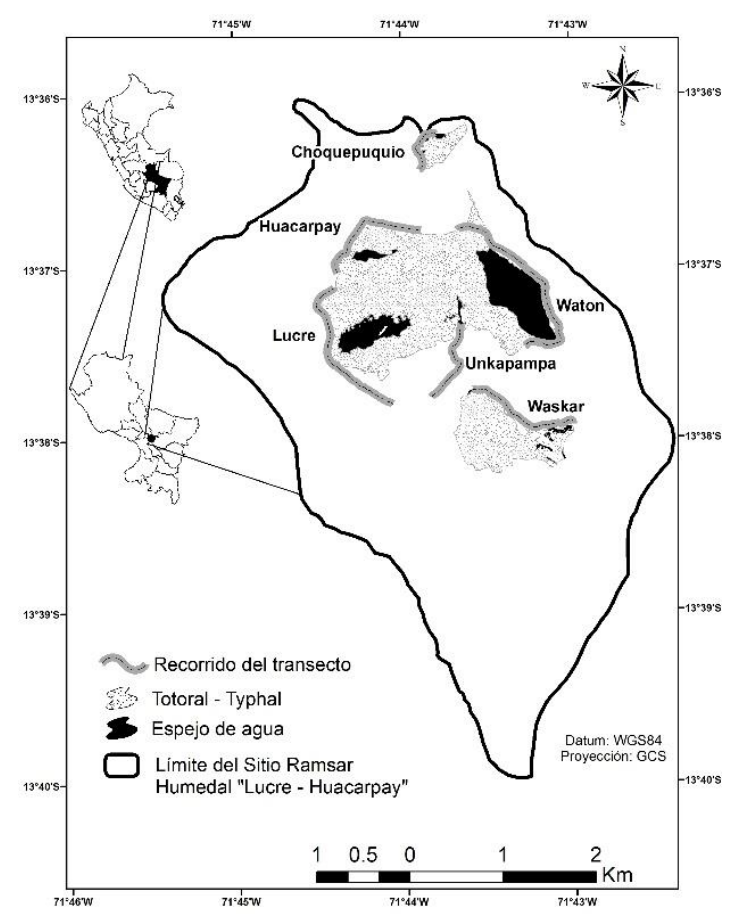

Figura 1. Mapa de ubicación del sitio Ramsar Humedal Lucre-Huacarpay, con los seis espejos de agua permanentes evaluados, localizados en la provincia de Quispicanchi, Región Cusco, Perú.

La vegetación del sitio Ramsar Humedal LucreHuacarpay tipifica como un bosque seco de valle interandino, donde se registraron 193 especies de plantas (Galiano et al., 2003; Galiano \& Nuñez, 2005). La vegetación del humedal en la actualidad está dominada por la asociación de Typha domingensis Pers. y Scirpus californicus var. tatora (Kunth) Barros, especies que se encuentran en una proporción similar. En cuanto a la vegetación circundante, Choquepuquio 
está conformada por bosques de Schinus molle L. y de Prosopis tupayachensis L. Vásquez, especie cuya distribución se restringe a 3 lugares de la Cuenca del Vilcanota (Venero et al., 2017), mientras que los otros espejos de agua salvo Unkapampa, se encuentran rodeados por parches de bosques de Schinus molle L. Sin embargo, la vegetación con mayor cobertura en el área circundante es el matorral seco espinoso, que está conformado por especies como Colletia spinosissima J.F. Gmel., Berberis boliviana Lechl., Puya ferruginea (Ruiz \& Pav.) L.B. Sm., Puya longistyla Mez, Opuntia ficus-indica (L.) Mill., Echinopsis cuzcoensis (Britton \& Rose) Friedrich \& G.D. Rowley, Dasyphyllum leiocephalum (Wedd.) Cabrera, Mutisia acuminata Ruiz \& Pav., Adesmia miraflorensis J. Remy, Austrocylindropuntia subulata (Muehlenpf.) Backeb., Corryocactus squarrosus (Vaupel) Hutchison, Corryocactus erectus (Backeb.) F. Ritter, Cylindropuntia tunicata (Lehm.) F.M. Knuth y otras. Toma de datos

Los muestreos se llevaron a cabo entre septiembre del 2015 y agosto del 2016. Las seis rutas de los espejos de agua que conforman el Humedal Lucre-Huacarpay (Figura 1), fueron visitados la quincena de cada mes puesto que en el área hay especies ocasionales y temporales que solo suceden en periodos cortos (Venero, 2015). Se utilizó el método de transectos (Ralph et al., 1996) los cuáles difirieron en longitud, porque se utilizaron las rutas de avistamiento de aves que se encuentran en el área, y se evaluaron a una velocidad constante aproximada de $1.5 \mathrm{~km} / \mathrm{hora}$. Se registraron todas las especies de aves encontradas a 150 metros hacia el humedal y 100 metros hacia al matorral, esta diferencia fue debido a la geografía del terreno. Las características del Humedal Lucre-Huacarpay son particulares, al ser un bosque seco que limita directamente con el humedal hace que algunas especies de aves e incluso mamíferos (Cavia tschudii) puedan desplazarse entre ambas unidades, por lo cual no se discriminó en métodos.

Las horas de avistamiento fueron en las mañanas de 7:00 am a 11:00 am. Para respetar los horarios establecidos, se dividió los sitios en dos grupos, el primero conformado por los espejos de agua de Lucre, Huacarpay y Choquepuquio, y el segundo por Watón, Unkapampa y Wascar; de manera que el segundo grupo fuera muestreado al día siguiente del primero. En cada espejo de agua se tardó en promedio una hora, el orden en que los sitios fueron muestreados se alternaba en cada evaluación. Para la observación y determinación de las especies de aves se utilizaron binoculares, cámaras fotográficas y guías de campo (Venero, 2008; Venero, 2015; Schulenberg et al., 2010; Walker, 2015). La nomenclatura y el orden taxonómico de las especies fueron tomadas de Plenge (2017). En cada sitio evaluado, se registró la especie de ave observada y el número de individuos. Adicionalmente, se registró el grado de residencia de las especies siguiendo a Venero
(2015), como Residente (R) a aquellas que están presentes todo el año, Migratorio (M), las que tienen un ciclo regular y periódico en el ámbito de estudio, Ocasional (O) las que son de registro único y Temporal (T) a las especies que no tienen regularidad alguna en la visita al humedal pero hay registros de la misma durante una temporada de duración variable. El estado de conservación de las especies fue obtenido de la categorización de especies amenazadas de fauna silvestre del Perú (Decreto Supremo No 004-2014-AG) (MINAGRI, 2014): en peligro (EN), vulnerable (VU), casi amenazado (NT) y preocupación menor (LC).

Análisis de datos

Se utilizaron los estimadores no paramétricos Chao2, Jacknife de primer orden y Jacknife de segundo orden para determinar la riqueza potencial del Humedal Lucre-Huacarpay en el periodo de evaluación. Para conocer la riqueza potencial tanto en los espejos de agua como en los 12 meses que duró el estudio, se utilizó el estimador no paramétrico Chao 1. La riqueza específica entre los espejos de agua se comparó utilizando curvas de rarefacción basadas en individuos (Moreno, 2001) que se usan cuando el esfuerzo de muestreo no fue igual.

Para estimar la estructura de la diversidad alfa se utilizaron el índice de dominancia de Simpson (1 - D) y el índice de equidad de Shannon-Wiener. Asimismo, se realizaron curvas de rango abundancia que describen la diversidad de la taxocenosis (Magurran, 1989) tanto para los seis espejos de agua como para las dos estaciones (secas y lluvias) y se compararon en función a la pendiente de la ecuación de la recta que se ajuste al modelo. La diversidad beta se calculó mediante el índice de similitud cualitativo de Jaccard y el índice de similitud cuantitativo de Morisita-Horn. Los diferentes índices de diversidad alfa y beta se realizaron en el programa PAST 3.16 (Hammer et al., 2001). La diferencia o igualdad de la diversidad en los distintos espejos de agua se calculó mediante el análisis de la varianza (ANOVA) para los valores del índice de Shannon-Wiener, donde las repeticiones constituyeron las evaluaciones mensuales. Para determinar los subgrupos homogéneos que se formaron, se utilizó la prueba posterior de Tukey-b.

Se realizó la prueba de T-Student para conocer si existe diferencia en la diversidad de Shannon-Wiener, número de individuos y número de especies producto de la estacionalidad, para lo cual se agruparon los meses considerados de época seca y los meses de lluvias. Para saber si existió una correlación significativa entre la longitud del transecto y el tamaño del espejo de agua con la diversidad de ShannonWiener, número de especies y el número de individuos, que podrían haber influido en el estudio, se realizó la prueba de correlación de Pearson. 


\section{Resultados}

$\underline{\text { Riqueza de especies }}$

Entre septiembre del 2015 y agosto del 2016, se registraron 6445 individuos de aves, distribuidos en 84 especies, 66 géneros, 29 familias y 15 órdenes. Las familias con mayor número de especies fueron Thraupidae con 11 especies, Tyrannidae con 10 especies, y Anatidae, Ardeidae y Scolopacidae con 6 especies cada una. De las especies determinadas según el grado de residencia, $63(75 \%)$ son residentes, 4 $(4.8 \%)$ son temporales, $5(6 \%)$ ocasionales y 12 (14.2\%) son migratorias (Tabla 1, en sección Apéndice). Respecto al estado de conservación 83 especies se encuentran en preocupación menor (LC) y 1 especie (Phoenicopterus chilensis) se encuentra casi amenazada (NT) (Tabla 1, en sección Apéndice). Las 5 especies con mayor abundancia fueron: Zonotrichia capensis (10.33\%), Chroicocephalus serranus $(9.46 \%)$, Fulica ardesiaca $(8.22 \%)$, Catamenia analis $(6.27 \%)$ y Gallinula galeata $(5.91 \%)$ que representan el $40.2 \%$ del total de individuos registrados. Se registraron 18 especies en todos los meses de evaluación. Los estimadores Chao 2, Jackknife de primer y segundo orden, indican que el número total de especies podría ser de 89, 95 y 98 especies respectivamente. Por lo que se logró registrar el 94.4\%, $88.4 \%$ y $85.7 \%$ del total de especies que podrían ocurrir en el periodo de 1 año, debido a que, de las 84 especies registradas, 8 fueron vistas una sola vez y 6 solo dos veces. Respecto a la abundancia porcentual de aves en relación a su residencia, se observó que en todos los meses las aves residentes representan más del $80 \%$ de las especies, incluso en meses donde ocurren aves migratorias, las cuales tuvieron su máxima representatividad en septiembre-2015 con un poco más del 10\% (Figura 2). Las curvas de rarefacción de los espejos de agua (Figura 3), para una muestra mínima de 752 individuos, indicaron que Waskar puede tener más de 50 especies, seguido por Waton, Lucre, Huacarpay y Choquepuquio, estos tres últimos para la muestra indicada, presentaron casi el mismo número de especies. Unkapampa sin embargo, es el espejo de agua que tuvo el menor número de especies (un poco más de $30)$.

Diversidad alfa

\section{De los espejos de agua}

El espejo de agua que tuvo la mayor riqueza de especies fue Waskar con 63 especies, seguido por Waton con 58, mientras que el menor número de especies se observó en Unkapampa con 43 especies (Figura 4). Asimismo, Waskar presentó los mayores valores del índice de Simpson, índice de ShannonWiener y Chao-1, que nos muestra una mayor equitatividad entre los individuos de cada especie. Unkapampa por otro lado, es el espejo de agua que presentó el menor valor de todos los índices de diversidad alfa, resaltando la dominancia de unas pocas especies, y la desproporción en la distribución de los individuos por especies. Según el índice de Chao 1, en Choquepuquio se podrían encontrar 60 especies, 53 especies en Huacarpay, 50 especies en Lucre, 45 especies en Unkapampa, 68 especies en Waskar, y 67 especies en Waton.

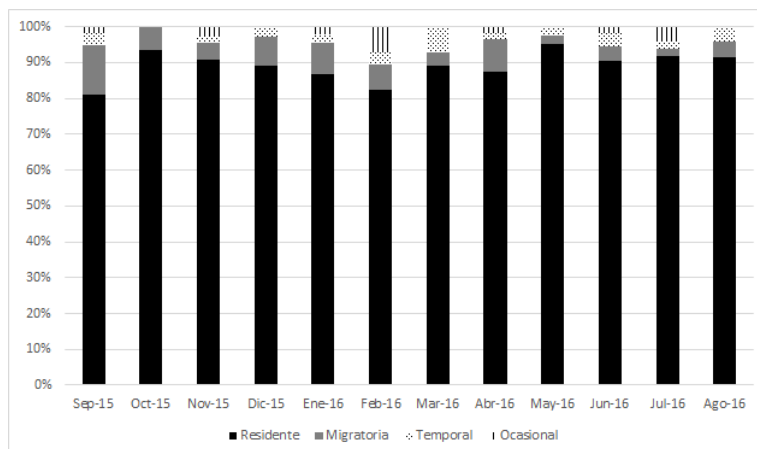

Figura 2. Estacionalidad porcentual de aves por meses.

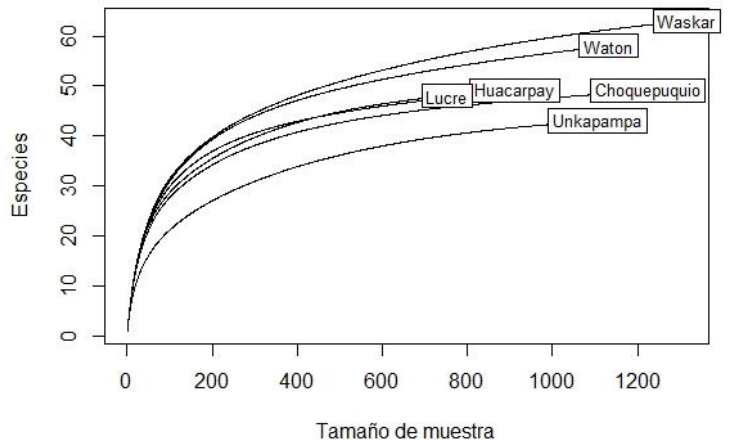

Figura 3. Curvas de rarefacción de los espejos de agua.

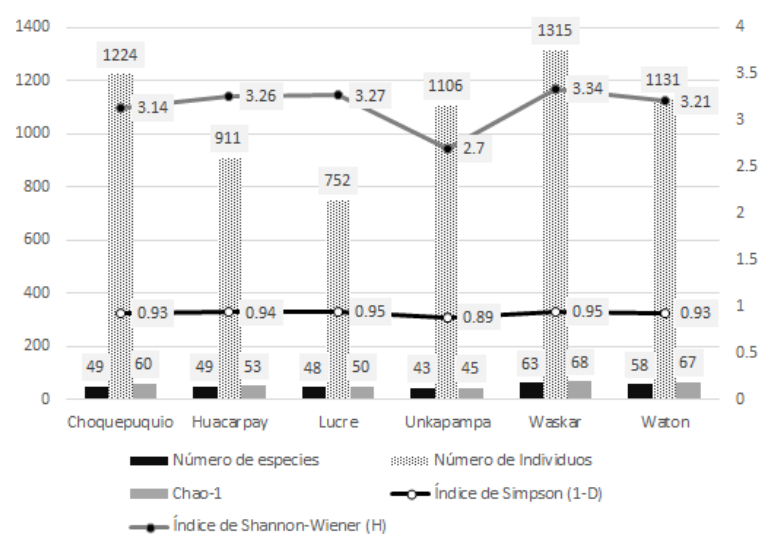

Figura 4. Índices de diversidad alfa de los espejos de agua.

\section{De los meses}

El mayor número de especies se observó en los meses de septiembre y febrero con 57 especies cada uno, mientras que el mayor número de individuos se registró en los meses de septiembre (801) y agosto (778) (Figura 5). En relación con los índices de diversidad alfa, los mayores valores del índice de Simpson se obtuvieron en enero y febrero, el mayor 


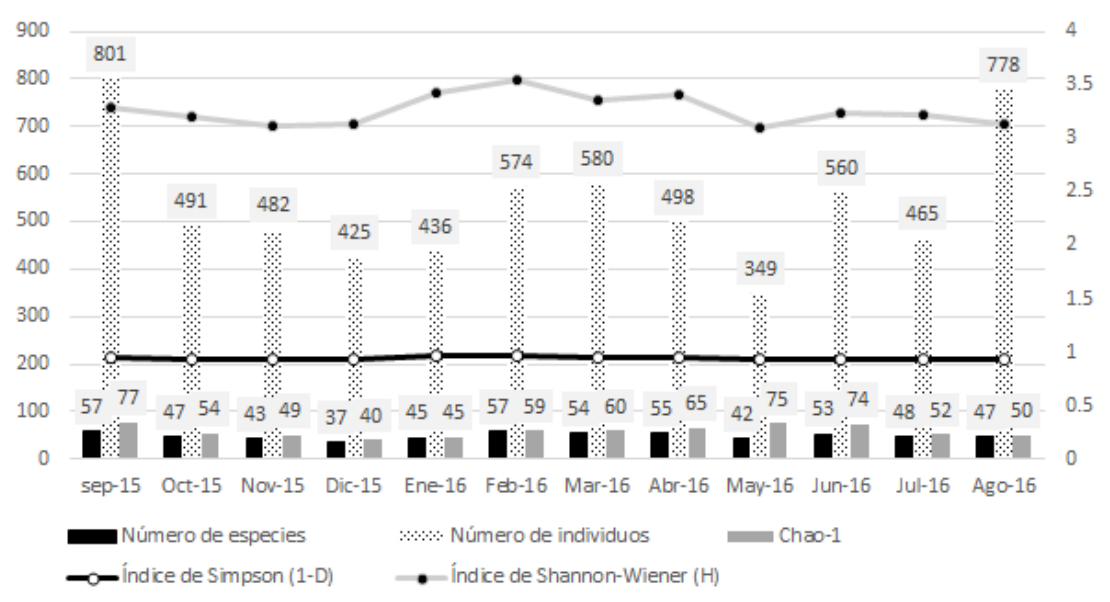

Figura 5. Índices de diversidad alfa de los meses.

valor del índice de Shannon-Wiener en febrero. Finalmente, el mayor número de especies que se podría registrar según el índice de Chao-1 se dio en septiembre (77 especies).

\section{$\underline{\text { Diversidad beta }}$}

\section{Similitud entre los espejos de agua}

La similitud de especies del índice cualitativo de Jaccard, muestra que los espejos de agua más parecidos son Waskar - Waton, Choquepuquio - Waskar y Choquepuquio - Waton, con índices de similitud del $68 \%, 67 \%$ y $65 \%$. La mayor disimilitud se dio entre Huacarpay - Waskar, que solo comparten un $45 \%$ de las especies.

Mientas que la similitud de la abundancia poblacional de aves del índice de Morisita-Horn, indicó que los cuerpos de agua más parecidos son: Lucre Waskar, Lucre - Waton y Waskar - Waton, con una similitud del $89 \%, 77 \%$ y $87 \%$, respectivamente. La mayor disimilitud se presentó entre Huacarpay - Waton $(37 \%)$.

\section{Similitud entre los meses}

La similitud de especies del índice de Jaccard indicó que los meses más parecidos fueron: jun-16 - jul-16, jun-16 - ago-16, mar-16 - abr-16 y feb-16 - mar-16, con similitudes del 77\%, 75\%, 73\% y 73\%, respectivamente. Mientras que los meses que presentaron menor similitud fueron: dic-15 - jul-16 (44\%).

El índice de similitud de Morisita-Horn, reveló que los meses con mayor similitud en la abundancia del total de especies fueron oct-15 - nov-15, nov-15 - dic15 y mar-16 - abr-16, con un $95 \%$, 95\% y $94 \%$ de similitud, respectivamente. Mientras que la mayor disimilitud fue entre los meses de may-16 - jul-16 (67\%).

Curvas de rango-abundancia

\section{De los espejos de agua}

Las curvas de rango-abundancia para los espejos de agua (Figura 6) presentan considerable dominancia de especies: Zonotrichia capensis en Lucre, Waskar y
Waton y Fulica ardesiaca en Unkapampa y Huacarpay. Respecto a Unkapampa, que presentó la curva con la mayor pendiente y el menor coeficiente de determinación (Tabla 2), se observó en la $3^{\text {ra }}$ posición de dominancia a la especie Patagioenas maculosa, especie que está asociada con áreas de cultivos y refleja la presión antrópica hacia el humedal, en la posición 18 de $43(18 / 43)$ de la curva se encuentra Oreonympha nobilis, especie endémica y de cotizado valor para los observadores de aves; encontrada en esta ruta puesto que existen sectores de plantas ruderales del género Nicotiana que son atractantes para esta especie (Venero, 2015). En Waskar, que tiene la menor pendiente y un buen ajuste a la ecuación de la recta (98\%); también se encontró a Oreonympha nobilis además de la segunda especie endémica Asthenes ottonis (24/63). La especie exótica Columba livia, fue registrada en Huacarpay (11/49) y Waton (16/58), ambos lugares limitan con el centro poblado de Huacarpay en donde medran individuos de esta especie.

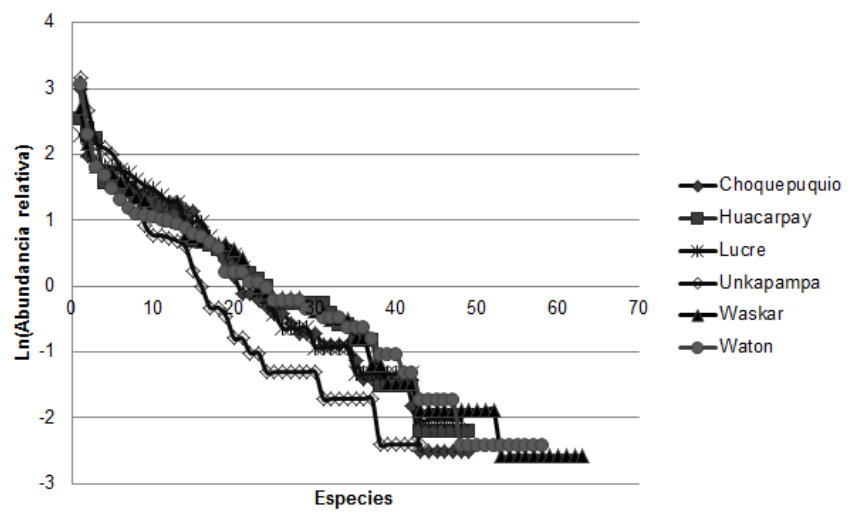

Figura 6. Curvas de rango abundancia para los 6 espejos de agua.

La mayor cantidad de especies migratorias fue registrada en el espejo de agua de Huacarpay y se ubican en relación a su abundancia relativa entre el $4^{\circ}$ y $3^{\text {er }}$ cuarto de la curva. Las colas uniformes en el 
último cuarto de la mayoría de las curvas, se pueden tratar de especies ocasionales o temporales como Tyrannus tyrannus, Phoenicopterus chilensis, Lophoneta specularoides y Diglossa sittoides o también especies residentes que tienen baja detectabilidad como Tachuris rubrigastra y Nothoprocta pentlandii. En resumen, las curvas de rango abundancia reflejan dominancia y perturbación, al encontrar especies exóticas y también generalistas como Zonotrichia capensis en posiciones destacadas, sin embargo, la pendiente entre las curvas no difiere demasiado, además de tener un ajuste considerablemente alto a la ecuación de la recta, salvo en Unkapampa, que aparte de tener la menor cantidad de especies posee la mayor pendiente y el menor ajuste (94\%) (Tabla 2), debido a la mayor dispersión de los datos.

\section{Por estacionalidad}

Las curvas de rango abundancia por estacionalidad (Figura 7) mostraron que en las 5 primeras posiciones de dominancia se repiten 4 especies en ambas épocas (Chroicocephalus serranus, Zonotrichia capensis, Fulica ardesiaca, y Catamenia analis), mientras que Gallinula galeata es reemplazada por Spatula puna en la época de secas. La cola más pronunciada de la estación de lluvias, en su mayoría representa a especies ocasionales, temporales y migratorias con una baja abundancia relativa. Las ecuaciones de las rectas ajustadas a los modelos (Figura 7) muestran poca diferencia en sus pendientes, así como un alto coeficiente de determinación $\left(\mathrm{R}^{2}\right)$. Por tanto, ambas curvas de rango abundancia representan estructuras similares en la comunidad al compartir el $70 \%$ de las especies.

Tabla 2. Ecuación de la recta y ajuste a las curvas de rango abundancia de los espejos de agua.

\begin{tabular}{llll}
\hline Espejo de agua & Ecuación de ajuste & $\begin{array}{l}\text { Coeficiente de } \\
\text { determinación }\end{array}$ & $\begin{array}{c}\text { : } \\
\text { Waskar }\end{array}$ \\
Waton & $\mathrm{y}=-0.0813 \mathrm{x}+2.05$ & $\mathrm{R}^{2}=0.98$ & -0.08 \\
Huacarpay & $\mathrm{y}=-0.083 \mathrm{x}+2.04$ & $\mathrm{R}^{2}=0.97$ & -0.08 \\
Lucre & $\mathrm{y}=-0.0934 \mathrm{x}+2.26$ & $\mathrm{R}^{2}=0.97$ & -0.09 \\
Choquepuquio & $\mathrm{y}=-0.1016 \mathrm{x}+2.31$ & $\mathrm{R}^{2}=0.97$ & -0.1 \\
Unkapampa & $\mathrm{y}=-0.1217 \mathrm{x}+2.24$ & $\mathrm{R}^{2}=0.94$ & -0.12 \\
\hline
\end{tabular}

Análisis de Varianza - ANOVA y T-Student.

El análisis de varianza del promedio de la diversidad de Shannon-Wiener de los espejos de agua indicó que existe diferencia estadística significativa de los lugares evaluados $(\mathrm{p}=0.00 ; \mathrm{GL}=5, \mathrm{Fcal}=19.13)$. Luego, la prueba de Tukey-b indicó que se formaron dos subgrupos homogéneos, el primero $(\mathrm{p}=1)$ representado solo por Unkapampa, y el segundo grupo homogéneo $(\mathrm{p}=0.346)$ conformado por Choquepuquio, Waskar, Waton, Lucre y Huacarpay (en orden del espejo de mayor promedio de la diversidad de Shannon-Wiener para los 12 meses).

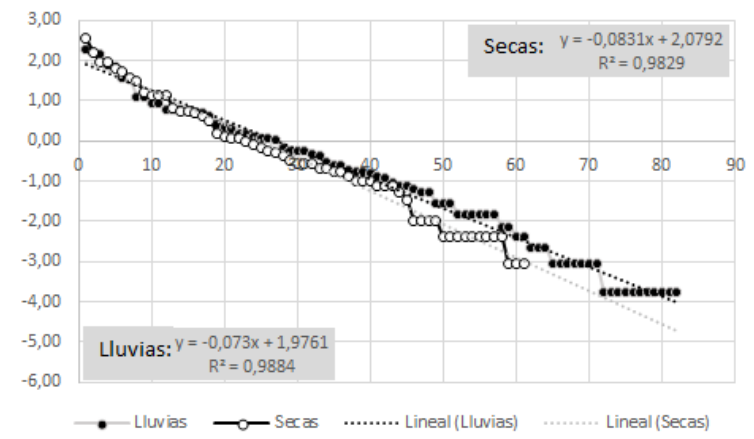

Figura 7. Curva de rango abundancia entre estaciones.

Entre tanto la prueba T-Student, muestra que no existe diferencia estadística significativa entre las medias de la diversidad de Shannon-Wiener, entre la época de lluvias y de secas ( $\mathrm{p}=0.061 ; \mathrm{GL}=10$; Tcal=2.115), tampoco en la riqueza de especies $(\mathrm{p}=0.657 ; \mathrm{GL}=10 ; \mathrm{Tcal}=0.457)$ ni en la abundancia de individuos $(\mathrm{p}=0.981 ; \mathrm{GL}=10$; $\mathrm{Tcal}=-0.024)$

Prueba de Correlación de Pearson

Respecto a la correlación entre la longitud del transecto con el índice de diversidad de ShannonWiener (Pearson=0.463, $\mathrm{P}=0.355$ ), el número de individuos (Pearson=-0.449, $\mathrm{P}=0.371$ ) o el número de especies (Pearson $=0.379, \mathrm{P}=0.458$ ) es estadísticamente no significativa, debido a que el valor de significancia es superior a 0.05 en todos los casos. Del mismo modo, no existe correlación significativa entre el área del espejo de agua con el índice de Shannon-Wiener (Pearson=0.236, $\mathrm{P}=0.653$ ), el número de individuos (Pearson=-0.194, $\mathrm{P}=0.713$ ) o el número de especies (Pearson=0.318, $\mathrm{P}=0.539$ ) al aceptarse la hipótesis nula en todos los casos.

\section{Discusión}

En el presente estudio se reportaron 84 especies de aves que representan aproximadamente un $4.5 \%$ de las 1852 especies reportadas para el Perú (Plenge, 2017) y el $60 \%$ de las 140 reportadas hasta el momento para el Humedal Lucre-Huacarpay (Venero, 2015). Sin embargo, el total de aves reportadas para el humedal son registros de varios años, mientras que los resultados presentados corresponden al periodo de un año completo de evaluación. Algunos de los registros durante este estudio son referidos a patrones atípicos para este humedal. Este es el caso de Phoenicopterus chilensis, que de acuerdo con Venero (2015) el último registro data del año 2011, de dos ejemplares inmaduros que se quedaron durante 3 semanas; en cambio, en el presente estudio se tuvo el registro de esta especie durante prácticamente todo el año; y el mayor número de individuos observado fue de 11 en el mes de 
junio. Al respecto, algunos autores (Ricalde, 2003; Martínez \& Morales, 2016) señalan que el frio intenso y heladas considerables en las alturas afectan los desplazamientos verticales de esta especie. Perrins (2011), en relación al tema, menciona que la distribución y conducta de Phoenicopterus chilensis es poco predecible por su comportamiento errático.

Otro registro a destacar es el de Phalacrocorax brasilianus y su permanencia durante todo el año de evaluación, además de un registro inusual de 22 y 10 individuos en enero y febrero, respectivamente. Al respecto, Venero (2015) asevera que solo ocurren solitarios o en parejas; para Guevara et al. (2012) los desplazamientos de esta especie son desconocidos y pueden deberse a cambios en el comportamiento, influencia demográfica y disponibilidad de alimentos. Así también, Rynchops niger, cuyo único reporte en el área fue en febrero del 2013 (Venero, 2015), fue observado en el presente estudio en el mismo mes del año 2016; para Schulenberg et al. (2010) esta es una especie divagante en el altiplano, mientras que Davenport et al. (2016) mencionan que sigue diferentes rutas transandinas para su migración desde la Amazonia hacia la costa del pacifico. De cualquier forma, se requiere más información sobre los patrones de movimiento de esta especie.

En lo referente a la riqueza de aves migratorias Venero (2015) registró 23 especies durante 15 años de trabajo de campo, de las cuales 12 fueron reportadas en este estudio; de ellas, algunas tuvieron un solo registro como Petrochelidon pyrrhonata, mientras que otras, como Tringa flavipes, fue registrada todo del año. Torres et al. (2006) explican que algunos individuos de especies migratorias retrasan o incluso no llegan a migrar debido a que no presentan las condiciones físicas adecuadas. Con la información del presente estudio no es posible aseverar si el número de aves migratorias registradas fue normal o atípico para el área debido a que no se tienen antecedentes comparables.

Respecto a los índices de diversidad alfa por ruta evaluada, Waskar tuvo los valores más altos, pero la prueba de Tukey-b indicó que es estadísticamente igual a Waton, Lucre, Huacarpay y Choquepuquio. Por otro lado, Unkapampa es el espejo de agua que presentó los valores más bajos de diversidad alfa, a pesar de que constituye un ecotono hídrico entre aguas lóticas y lénticas (Venero, 2015). Tukey-b reveló que esta diversidad es estadísticamente inferior a los demás espejos de agua. Para tener una respuesta concluyente acerca de la causa de la baja diversidad, se sugiere realizar más estudios complementarios que consideren otras variables como el efecto del uso del suelo, cobertura vegetal y presencia de especies exóticas.

La diversidad de Shannon-Wiener más alta se observó en febrero del 2016. Este resultado es comparable al de Iannacone et al. (2010) para Pantanos de Villa en el año 2006; en consecuencia, la mayor proporcionalidad de individuos por especies se dio en febrero, tanto para dicho humedal costero como para el humedal andino evaluado en el presente trabajo. Sin embargo, no informa mucho acerca de la estructura de la comunidad de aves en ambos ecosistemas.

A pesar que el índice cualitativo de Jaccard mostró la mayor disimilitud entre meses de lluvias y secas, al agrupar los meses, ambas estaciones comparten el 70\% de especies; de las 61 especies que ocurren en la época de secas, 59 fueron registradas también en la época de lluvias. La prueba T-Student reveló que no hay diferencia significativa de la diversidad entre estaciones para este humedal. De igual forma, Iannacone et al. (2010), resaltaron la no diferencia estacional para Pantanos de Villa; sin embargo, en su trabajo del humedal costero, las aves migratorias representaron entre el 5.88 y $47.82 \%$ del total de especies, mientras que en el Humedal Lucre-Huacarpay solamente representaron el $14.2 \%$ del total de registros y con bajas abundancias relativas. Venero (2015) resalta que muchas especies consideradas migratorias altitudinales en otros humedales (Pulido, 2018) son residentes para el Humedal Lucre-Huacarpay, debido a que es considerado como un ecotono altitudinal.

Establecido que, ni la longitud del transecto ni el área del espejo de agua tuvieron una correlación significativa con la diversidad y riqueza de especies de aves en los espejos de agua, es importante analizar otras variables no presentadas en el estudio. Se debe continuar con estudios que cuantifiquen a las poblaciones de aves en estaciones de evaluación permanente que permitan comparaciones en el tiempo.

Con los datos obtenidos no es posible aseverar si el fenómeno de El Niño ocurrido en el periodo de evaluación tuvo una influencia significativa en la dinámica de las poblaciones del humedal. Lo que se resalta es que algunos lugares, que suelen tener inundación temporal, no presentaron tal característica debido a las escasas precipitaciones que, podrían estar relacionadas con el impacto del fenómeno de El Niño en el periodo de evaluación (Martínez et al., 2017).

Por lo encontrado en el presente trabajo en referencia a la diversidad, el Humedal LucreHuacarpay es de importancia para actividades ambientalmente sostenibles como la observación de aves y el ecoturismo (Walker, 2015) durante todo el año, al ser un refugio de vida silvestre reconocido como sitio Ramsar.

Finalmente, destacamos que la pérdida del hábitat natural es la principal amenaza para la disminución de diversidad de aves a nivel mundial (Myers et al., 2000) y este hecho no es ajeno al Humedal Lucre-Huacarpay, cuyas principales amenazas son el cambio en el uso de suelo, flora y fauna exótica.

\section{Conclusiones}

El presente estudio registró 84 especies de aves, en el Humedal Lucre-Huacarpay, para el periodo de septiembre del 2015 a agosto del 2016. Los meses con 
mayor diversidad y riqueza de especies fueron febrero2016 y septiembre-2015; sin embargo, no se presentó diferencia significativa entre las estaciones de secas y lluvias, debido a que ambos periodos comparten el $70 \%$ de las especies.

Waskar tuvo los valores más altos de diversidad, teniendo la mejor proporción de individuos por especies, pero es estadísticamente igual a Waton, Huacarpay, Lucre y Choquepuquio; mientras que Unkapampa presentó los valores más bajos, además de la curva de rango abundancia con el menor ajuste a la ecuación de la recta y de menor tamaño, indicando dominancia y menor número de especies.

En este humedal se presentan especies temporales y ocasionales, razón por la cuál es necesario continuar con evaluaciones periódicas de la diversidad y riqueza de especies. Asimismo, se sugiere realizar una evaluación similar a la presentada, en un periodo donde el fenómeno de El Niño no esté presente, para poder establecer comparaciones.

Los estudios a largo plazo en el humedal respaldarán el mantenimiento del hábitat del cual depende la avifauna, además de garantizar y fortalecer las actividades turísticas en el tiempo.

\section{Agradecimientos}

Al Dr. José Luis Venero Gonzales, por su colaboración en las salidas de campo y la revisión del artículo. Así también, a los revisores de la Revista Aplicada de Ecología, quienes ayudaron a mejorar la presentación del trabajo sustancialmente.

\section{Literatura citada}

Apaza M. \& Figari A. 1999. Mortandad de aves marinas durante "El Niño 1997 - 98" en el litoral sur de San Juan de Marcona, Ica - Perú. Rev. Peru. Biol. 6(3): 110 - 117; número extraordinario ("El Niño 1997-98 y su impacto sobre los ecosistemas marino y terrestre").

Bildstein K., Bancroft G., Dugan P., Gordon D., Edwin R., Noel E., Payne L. \& Senner S. 1991. Approaches to the conservation of coastal wetlands in the Western Hemisphere. Wilson Bulletin 103(2): 218-254. URL: https://sora.unm.edu/node/130909.

Blanco D.1999. Los Humedales como hábitat de aves acuáticas. En: Malvárez A.I. (Ed.). 1999. Tópicos sobre humedales subtropicales y templados de Sudamérica, pp: 219-228. ORCYT-UNESCO, Montevideo, Uruguay.

Cronk J.K. \& Fennessy M.S. 2016. Wetland plants: biology and ecology. CRC press.

Davenport L.C., Goodenough K.S. \& Haugaasen T. 2016. Birds of Two Oceans? Trans-Andean and Divergent Migration of Black Skimmers (Rynchops niger cinerascens) from the Peruvian Amazon. PLoS ONE11(1): e0144994. DOI: https://doi.org/10.1371/journal.pone.0144994.

Galiano W. \& Nuñez P. 2005. Wakarpay: relicto de bosque seco en el valle del Cusco. En: Historia Natural del Valle del Cusco. pp: 251-262. SOPRONAC, Cusco, Perú.

Galiano W., Nuñez P. \& Alvarez E. 2003. Huacarpay: relicto de bosque seco de valle interandino: inventario florístico de un ecosistema en peligro. Cantua (12): 27-33.
Gauthier G., Giroux J., Reed A., Beéchet A. \& Bélanger L. 2005. Interactions between land use, habitat use, and population increase in greater snow geese: what are the consequences for natural wetlands? Global Change Biology 11: 856-868. DOI: https://doi.org/10.1111/j.1365-2486.2005.00944.x.

Giraldo A., Véliz C., Arellano G. \& Sánchez E. 2002. El uso de la Ley de Taylor en el establecimiento de patrones de variación espacio-temporal en poblaciones animales: dos ejemplos de aplicación. Ecología Aplicada, 1: 71-74. DOI: http://dx.doi.org/10.21704/rea.v1i1-2.232.

Guevara E., Santander T., Mueces T., Terán K. \& Pierre-Yves H. 2012. Population Growth and Seasonal Abundance of the Neotropic Cormorant (Phalacrocorax brasilianus) at Highland Lakes in Ecuator. Waterbirds, 34(4): 499-503.

Hammer Ø., Harper D.A.T., Ryan P.D. 2001. PAST: Paleontological statistics software package for education and data analysis. Palaeontologia Electronica 4(1): 1-9. URL: $\quad$ http://palaeoelectronica.org/2001 1/past/issue1 01.htm. PDF: https://palaeo-electronica.org/2001_1/past/past.pdf.

Iannacone J., Atasi M., Bocanegra T., Camacho M., Montes A., Santos S., Zuñiga H. \& Alayo M. 2010. Diversidad de aves en el humedal Pantanos de Villa, Lima, Perú: periodo 2004 - 2007. Biot. Neotrop., 10(2): 295-304. http://www.biotaneotropica.org.br/v10n2/en/abstract?inv entory+bn02610022010.

Jiménez-Muñoz J.C., Mattar C., Barichivich J., SantamariaArtigas A., Takahashi K., Malhi Y., Sobrino J.A., \& Van der Schrier G. 2016. Record-breaking warming and extreme drought in the Amazon rainforest during the course of El Niño 2015 - 2016. Scientific Reports, 6: 33130. DOI: https://doi.org/10.1038/srep33130.

Lyons J. \& Haig S. 1995. Fat content and stopover ecology of spring migrant semipalmated sandpipers in South Carolina. The Condor. 97(2): 427-437. URL: https://sora.unm.edu/sites/default/files/journals/condor/v 097n02/p0427-p0437.pdf.

Magurran A.E. 1989. Diversidad ecológica y su medición. Ediciones Vedrà. Barcelona.

Martínez O. \& A. Morales. 2016. Primer registro del flamenco "Tokoko" (Phoenicopterus chilensis) en el valle de la ciudad de La Paz: Laguna de Achocalla: Kempffiana, 12(2): 39-47.

Martínez R., Zambrano E., Nieto J.J., Hernández J. \& Costa F. 2017. Evolución, Vulnerabilidad e impactos económicos y sociales de El Niño 2015 - 2016 en América Latina. Investigaciones Geográficas, (68): 65 78. DOI: 10.14198/INGEO2017.68.04. URL: http://rua.ua.es/dspace/handle/10045/72010.

MINAGRI (Ministerio de Agricultura). 2014. Decreto Supremo que aprueba la actualización de la lista de clasificación y categorización de las especies amenazadas de fauna silvestre legalmente protegidas. Decreto Supremo No 004-2014-MINAGRI. El Peruano, martes 8 de abril de 2014, NORMAS LEGALES, Año XXXI - $\mathrm{N}^{\circ}$ 12813: 520497- 520504. Perú.

Moreno C. 2001. Métodos para medir la diversidad. M\&T GORFI S.A. Zaragoza, España.

Myers N., Mittermeier R. A., Mittermeier C.G., Da Fronseca G.A.B. \& Kent J. 2000. Biodiversity Hotspots for conservation priorities. Nature, 403: 853-858.

Perrins C. 2011. Enciclopedia completa de las aves. Editorial LIBSA. Madrid. 
Plenge M. 2017. Lista de las Aves de Perú. Lima, Perú. Disponible https://sites.google.com/site/boletinunop/checklist.

Posada-Marín J.A., Rendón A.M., Salazar J.F., Mejía J.F. \& Villegas J.C. 2019. WRF downscaling improves ERAInterim representation of precipitation around a tropical Andean valley during El Niño: implications for GCMscale simulation of precipitation over complex terrain. Climate Dynamics, 52(5-6): 3609-3629. DOI: https://doi.org/10.1007/s00382-018-4403-0.

Pulido V. \& Dourojeanni M. 1990. Variación estacional de las poblaciones de aves en las Lagunas de Mejía. Revista Forestal del Perú. 17(2): 49-69.

Pulido V. 2018. Estacionalidad de las especies de aves residentes y migratorias altoandinas en el lado peruano de la cuenca del Titicaca. Revista de Investigaciones Altoandinas, 20(4): 461-476. DOI: http://dx.doi.org/10.18271/ria.2018.423.

Ralph J.C., Geupel G.R., Pyle P., Martin T.E., De Sante D.F. \& Mila B. 1996. Manual de métodos de campo para el monitoreo de aves terrestres. United Stated Department of Agriculture, Pacific Southwest Research Station Albany, California.

RAMSAR (THE RAMSAR CONVENTION). 1971. Convention on Wetlands of International Importance especially as Waterfowl Habitat. Ramsar, Iran, 2.2.1971; as amended by the Protocol of París, 3.12.1982 and the Amendments of Regina, 28.5.1987. París, 13.7.94, Office of International Standards and Legal Affairs, United Nations Educational, Sicientific and Cultural Organization (UNESCO) https://www.ramsar.org/sites/default/files/documents/lib rary/scan certified e.pdf.

Ricalde D.G. 2003. Conservación de Flamencos en el altiplano peruano. Lyonia, 4(1): 87-96.

Schulenberg T.S., Stotz D.F., Lane D.F., O'neill J.P. \& Parker III T.A. 2010. Aves del Perú. Lima, Perú: Centro de Ornitología y Biodiversidad - CORBIDI.
Stewart R.Jr. 1996. Wetlands as Bird habitat. In: U.S. Geological Survey Water-Supply. 1996. National Water Summary on Wetlands Resources. Paper 2425. URL: https://water.usgs.gov/nwsum/WSP2425/birdhabitat.htm 1.

Suarez-Ognio L., Estela-Ayamamani D., Cáceres-Mejía B., Gambirazio-Carbajal C. \& Cabrera R. 2015. Impacto del fenómeno "El Niño" de 1997 - 1998 en la salud de la población peruana, riesgo potencial para el 2015. Rev. Peru. Med. Exp. y Salud Pública, 32(2): 395-406.

Torres M., Quinteros Z. \& Takano F. 2006. Variación temporal de la abundancia y diversidad de aves limícolas en el refugio de vida silvestre Pantanos de Villa, Lima Perú. Ecología Aplicada, 5: 119-125. DOI: http://dx.doi.org/10.21704/rea.v5i1-2.325

Tovar H. \& García L. 1982. Las poblaciones de aves guaneras durante "El Niño" de 1957. Boletín de Lima. 22: 34 - 46.

Venero J.L. 2008. Etnornitología y guía de aves en el Humedal Lucre-Huacarpay. Editorial Moderna. Cusco, Perú.

Venero J.L. 2015. Guía de Macrofauna y Etnornitología en "Lucre-Huacarpay". Editorial Moderna. Cusco, Perú.

Venero J.L., Hurtado L.B. \& Cárdenas W.N. 2017. Situación actual de Prosopis tupayachensis L. Vásquez, Escurra \& A. Huamán (Fabaceae), el "thacco" o algarrobo andino. Chloris chilensis, Año $20 \quad \mathrm{~N}^{\circ} 1$. URL: http://www.chlorischile.cl/portada36\%20(20-1).html.

Walker B. 2015. Field Guide to the Birds of Machu Picchu and the Cusco Region, Peru. Including a bird finding guide to the area. Lynx Editions. Barcelona, España.

WorldClim - Global Climate Data. 2016. Free climate data for ecological modeling and SIG. Recuperado de: http://www.worldclim.org/.

World Resources Institute. 2005. Los Ecosistemas y el Bienestar Humano: Humedales y Agua. Informe de Síntesis, Evaluación de los Ecosistemas del Milenio. Washington, DC, Estados Unidos (de América). 


\section{Apéndice}

Tabla 1. Lista de aves registradas en el sitio Ramsar Humedal Lucre-Huacarpay.

Clasificación taxonómica según Plenge (2017). NB = especies que ocurren regularmente en Perú, pero solo en su período no reproductivo, según Plenge (2017). Grado de residencia según Venero (2015): R: Residente, M: Migratoria, T: Temporal, O: Ocasional. Estado de conservación según Decreto Supremo No 004-2014-MINAGRI (MINAGRI, 2014): EN: En peligro. VU: Vulnerable. NT. Casi amenazado. LC: Preocupación menor.

\section{ORDENIFamiliaWombre científico}

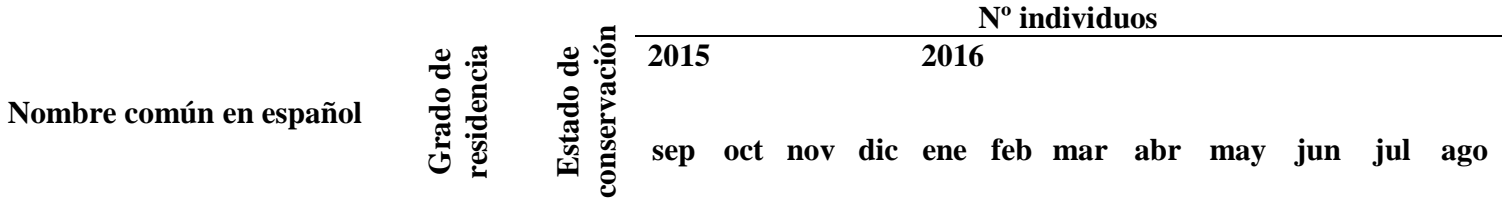

\section{TINAMIFORMES}

\section{Tinamidae}

Nothoprocta pentlandii Gray, 1867

\section{ANSERIFORMES}

\section{Anatidae}

Lophonetta specularioides King, 1828

Anas flavirostris Vieillot, 1816

Anas georgica Gmelin, 1789

Spatula puna Tschudi, 1844

Spatula cyanoptera Vieillot, 1816

Oxyura jamaicensis Gmelin, 1789

\section{PODICIPEDIFORMES}

Podicipedidae

Rollandia rolland Quoy \& Gaimard, 1824

\section{PHOENICOPTERIFORMES}

Phoenicopteridae

Phoenicopterus chilensis Molina, 1782

\section{SULIFORMES}

\section{Phalacrocoracidae}

Phalacrocorax brasilianus Gmelin, 1789

\section{PELECANIFORMES}

\section{Ardeidae}

Nycticorax nycticorax Linnaeus, 1758

Butorides striata Linnaeus, 1758

Bubulcus ibis Linnaeus, 1758

Ardea alba Linnaeus, 1758

Egretta thula Molina, 1782

Egretta caerulea Linnaeus, 1758

\section{Perdices}

Perdiz Andina

\section{Patos}

Pato Crestón

Pato Barcino

Pato Jergón

Pato de la Puna

Pato Colorado

Pato Rana

\section{Zambullidores}

Zambullidor Pimpollo

Flamengos

Flamenco Chileno

Cormoranes

Cormorán Neotropical

\section{Garzas}

Huaco Común

Garcita Estriada

Garcita Bueyera

Garza Grande

Garcita Blanca

Garcita Azul
$\mathrm{R}$

LC

$\mathrm{R}$

LC

$\begin{array}{cccccccccccccc}\mathrm{LC} & 0 & 0 & 0 & 0 & 0 & 0 & 0 & 0 & 0 & 1 & 0 & 1 & 0.03\end{array}$

$\begin{array}{llllllllllllll}\text { LC } & 21 & 13 & 25 & 9 & 21 & 11 & 12 & 17 & 11 & 10 & 17 & 33 & 3.10\end{array}$

$\begin{array}{llllllllllllll}\mathrm{LC} & 21 & 7 & 14 & 14 & 8 & 12 & 9 & 23 & 5 & 13 & 8 & 10 & 2.23\end{array}$

$\begin{array}{llllllllllllll}\mathrm{LC} & 16 & 17 & 19 & 8 & 5 & 10 & 7 & 5 & 4 & 24 & 40 & 88 & 3.77\end{array}$

$\begin{array}{llllllllllllll}\text { LC } & 17 & 8 & 4 & 8 & 12 & 1 & 3 & 7 & 1 & 3 & 7 & 34 & 1.63\end{array}$

$\begin{array}{llllllllllllll}\mathrm{LC} & 2 & 3 & 0 & 0 & 2 & 4 & 1 & 2 & 1 & 0 & 0 & 0 & 0.23\end{array}$

$\begin{array}{llllllllllllll}\mathrm{LC} & 3 & 0 & 4 & 5 & 4 & 4 & 3 & 0 & 4 & 1 & 2 & 3 & 0.51\end{array}$

$\begin{array}{llllllllllllll}\text { NT } & 0 & 0 & 0 & 0 & 3 & 2 & 0 & 0 & 0 & 11 & 2 & 0 & 0.28\end{array}$

$\begin{array}{llllllllllllll}\mathrm{LC} & 1 & 0 & 4 & 2 & 22 & 10 & 5 & 7 & 1 & 1 & 5 & 1 & 0.92\end{array}$

$\begin{array}{llllllllllllll}\mathrm{LC} & 0 & 2 & 0 & 0 & 0 & 0 & 0 & 0 & 0 & 0 & 1 & 2 & 0.08\end{array}$

$\begin{array}{llllllllllllll}\mathrm{LC} & 0 & 0 & 0 & 0 & 2 & 1 & 0 & 0 & 0 & 0 & 0 & 0 & 0.05\end{array}$

$\begin{array}{llllllllllllll}\mathrm{LC} & 18 & 13 & 7 & 21 & 7 & 6 & 9 & 12 & 11 & 3 & 0 & 7 & 1.77\end{array}$

$\begin{array}{llllllllllllll}\mathrm{LC} & 3 & 1 & 0 & 2 & 5 & 4 & 3 & 1 & 0 & 0 & 0 & 0 & 0.29\end{array}$

$\begin{array}{llllllllllllll}\mathrm{LC} & 0 & 3 & 0 & 0 & 3 & 0 & 0 & 1 & 0 & 0 & 3 & 0 & 0.16\end{array}$ 
Tabla 1. Lista de aves registradas en el sitio Ramsar Humedal Lucre-Huacarpay.

Clasificación taxonómica según Plenge (2017). NB = especies que ocurren regularmente en Perú, pero solo en su período no reproductivo, según Plenge (2017). Grado de residencia según Venero (2015): R: Residente, M: Migratoria, T: Temporal, O: Ocasional. Estado de conservación según Decreto Supremo Nº 004-2014-MINAGRI (MINAGRI, 2014): EN: En peligro. VU: Vulnerable. NT. Casi amenazado. LC: Preocupación menor.

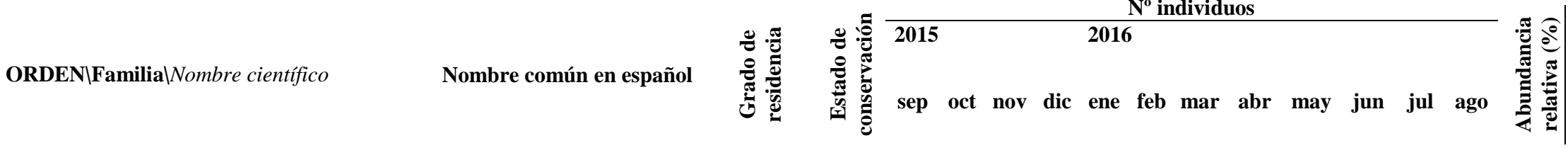

\begin{tabular}{|c|c|}
\hline Threskiornithidae & Ibis y espátulas \\
\hline Plegadis ridgwayi Allen, 1876 & Ibis de la Puna \\
\hline \multicolumn{2}{|l|}{ ACCIPITRIFORMES } \\
\hline Accipitridae & Gavilanes y águilas \\
\hline Circus cinereus Vieillot, 1816 & Aguilucho Cenizo \\
\hline Geranoaetus polyosoma Quoy \& Gaimard, 1824 & Aguilucho Variable \\
\hline Geranoaetus melanoleucus Vieillot, 1819 & Aguilucho de Pecho Negro \\
\hline \multicolumn{2}{|l|}{ GRUIFORMES } \\
\hline Rallidae & Rascones y gallaretas \\
\hline Pardirallus sanguinolentus Swainson, 1838 & Rascón Plomizo \\
\hline Gallinula galeata Lichtenstein, 1818 & Polla de agua \\
\hline Fulica ardesiaca Tschudi, 1843 & Gallareta Andina \\
\hline \multicolumn{2}{|l|}{ CHARADRIIFORMES } \\
\hline Charadriidae & Chorlitos y avefrías \\
\hline Pluvialis dominica Müller, 1776 (NB) & Chorlo Dorado Americano \\
\hline Vanellus resplendens Tschudi, 1843 & Avefría Andina \\
\hline Recurvirostridae & Avocetas y cigueñelas \\
\hline Himantopus mexicanus Linnaeus, 1758 & Cigüeñuela de Cuello Negro \\
\hline Scolopacidae & Correlimos y agachadizas \\
\hline Calidris bairdi Coues, 1861(NB) & Playerito de Baird \\
\hline Calidris melanotos Vieillot, 1819 (NB) & Playero Pectoral \\
\hline Phalaropus tricolor Vieillot, 1819 (NB) & Faláropo Tricolor \\
\hline Tringa solitaria Wilson, 1813 (NB) & Playero Solitario \\
\hline Tringa melanoleuca Gmelin, 1789 (NB) & Playero Pata Amarilla Mayor \\
\hline Tringa flavipes Gmelin, 1789 (NB) & Playero Pata Amarilla Menor \\
\hline Laridae & Gaviotas \\
\hline Chroicocephalus serranus Tschudi, 1844 & Gaviota Andina \\
\hline Rhynchopidae & Rayadores \\
\hline Rynchops niger Linnaeus, 1758 & Rayador Negro \\
\hline
\end{tabular}

$\begin{array}{lcccccccccccccc}\mathrm{R} & \mathrm{LC} & 61 & 14 & 31 & 29 & 21 & 8 & 12 & 21 & 21 & 62 & 9 & 28 & 4.92 \\ & & & & & & & & & & & & & & \\ \mathrm{R} & \mathrm{LC} & 1 & 0 & 0 & 0 & 0 & 0 & 1 & 1 & 0 & 5 & 1 & 2 & 0.17 \\ \mathrm{R} & \mathrm{LC} & 1 & 0 & 1 & 0 & 0 & 0 & 0 & 0 & 0 & 0 & 0 & 0 & 0.03 \\ \mathrm{R} & \mathrm{LC} & 1 & 1 & 0 & 0 & 0 & 0 & 0 & 0 & 1 & 0 & 1 & 0 & 0.06 \\ & & & & & & & & & & & & & & \\ \mathrm{R} & \mathrm{LC} & 19 & 8 & 9 & 12 & 12 & 9 & 14 & 11 & 9 & 13 & 14 & 13 & 2.22 \\ \mathrm{R} & \mathrm{LC} & 63 & 41 & 35 & 30 & 20 & 16 & 33 & 40 & 29 & 23 & 20 & 31 & 5.91 \\ \mathrm{R} & \mathrm{LC} & 65 & 62 & 50 & 49 & 32 & 30 & 49 & 39 & 32 & 26 & 19 & 77 & 8.22\end{array}$

Rayador Negro

$\begin{array}{lcccccccccccccc}\mathrm{M} & \mathrm{LC} & 1 & 0 & 0 & 0 & 0 & 0 & 0 & 0 & 0 & 0 & 0 & 0 & 0.02 \\ \mathrm{R} & \mathrm{LC} & 18 & 5 & 3 & 4 & 0 & 2 & 0 & 2 & 4 & 5 & 7 & 7 & 0.88 \\ \mathrm{M} & \mathrm{LC} & 0 & 0 & 2 & 6 & 6 & 16 & 0 & 0 & 0 & 0 & 0 & 0 & 0.47 \\ \mathrm{M} & \mathrm{LC} & 3 & 0 & 0 & 0 & 0 & 0 & 0 & 1 & 0 & 0 & 0 & 0 & 0.06 \\ \mathrm{M} & \mathrm{LC} & 2 & 0 & 0 & 0 & 0 & 0 & 0 & 0 & 0 & 0 & 0 & 0 & 0.03 \\ \mathrm{M} & \mathrm{LC} & 6 & 0 & 0 & 0 & 0 & 0 & 0 & 1 & 0 & 0 & 0 & 0 & 0.11 \\ \mathrm{M} & \mathrm{LC} & 2 & 1 & 0 & 0 & 0 & 0 & 0 & 0 & 0 & 0 & 0 & 0 & 0.05 \\ \mathrm{M} & \mathrm{LC} & 1 & 1 & 0 & 2 & 0 & 1 & 3 & 1 & 0 & 0 & 0 & 0 & 0.14 \\ \mathrm{M} & \mathrm{LC} & 2 & 0 & 0 & 1 & 4 & 9 & 22 & 6 & 2 & 2 & 0 & 3 & 0.79 \\ & & & & & & & & & & & & & & \\ \mathrm{R} & \mathrm{LC} & 58 & 47 & 55 & 45 & 40 & 58 & 56 & 53 & 14 & 57 & 78 & 49 & 9.46 \\ \mathrm{O} & \mathrm{LC} & 0 & 0 & 0 & 0 & 0 & 1 & 0 & 0 & 0 & 0 & 0 & 0 & 0.02\end{array}$


Tabla 1. Lista de aves registradas en el sitio Ramsar Humedal Lucre-Huacarpay.

Clasificación taxonómica según Plenge (2017). NB = especies que ocurren regularmente en Perú, pero solo en su período no reproductivo, según Plenge (2017). Grado de residencia según Venero (2015): R: Residente, M: Migratoria, T: Temporal, O: Ocasional. Estado de conservación según Decreto Supremo Nº 004-2014-MINAGRI (MINAGRI, 2014): EN: En peligro. VU: Vulnerable. NT. Casi amenazado. LC: Preocupación menor.

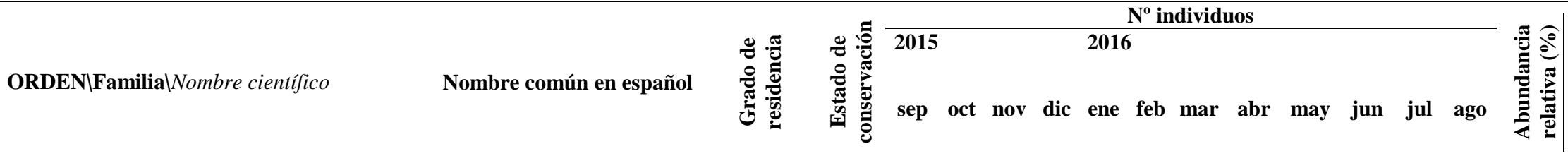

\author{
COLUMBIFORMES \\ Columbidae \\ Columba livia Gmelin, 1789 (IN) \\ Patagioenas maculosa Temminck, 1813 \\ Zenaida auriculata Des Murs, 1847 \\ Metriopelia ceciliae Lesson, 1845 \\ Metriopelia melanoptera Molina, 1782

\section{STRIGIFORMES} \\ Strigidae \\ Glaucidium peruanum König, 1991

\section{APODIFORMES} \\ Apodidae \\ Streptoprocne zonaris Shaw, 1796 \\ Trochilidae \\ Colibri coruscans Gould, 1846 \\ Lesbia victoriae (Bourcier \& Mulsant, 1846 \\ Lesbia nuna Lesson, 1832 \\ Oreonympha nobilis Gould, 1869 (E) \\ Patagona gigas Vieillot, 1824

\section{PICIFORMES} \\ Picidae \\ Colaptes rupícola d'Orbigny, 1840

\section{FALCONIFORMES}

\section{Falconidae} \\ Phalcoboenus megalopterus Meyen, 1834 \\ Falco sparverius Linnaeus, 1758 \\ Falco femoralis Temminck, 1822 \\ PASSERIFORMES \\ Furnariidae \\ Phleocryptes melanops Vieillot, 1817
}

\section{Palomas}

Paloma Doméstica

Paloma de Ala Moteada

Tórtola Orejuda

Tortolita Motea

Tortolita de Ala Negra

\section{Búhos y lechuzas}

Lechucita Peruana

Vencejos

Vencejo de Collar Blanco

\section{Picaflores}

Oreja-Violeta de Vientre

Azul

Colibrí de Cola Larga Negra

Colibrí de Cola Larga Verde

Montañes Barbudo

Colibrí Gigante

\section{Carpinteros}

Carpintero Andino

\section{Halcones}

Caracara Cordillerano

Cernícalo Americano

Halcón Aplomado

\section{Horneros}

Junquero

$\begin{array}{lcccccccccccccc}\mathrm{R} & \mathrm{LC} & 13 & 5 & 1 & 7 & 7 & 5 & 1 & 7 & 1 & 3 & 5 & 0 & 0.85 \\ \mathrm{R} & \mathrm{LC} & 36 & 2 & 1 & 0 & 7 & 19 & 4 & 10 & 4 & 9 & 30 & 2 & 1.92 \\ \mathrm{R} & \mathrm{LC} & 13 & 3 & 10 & 4 & 4 & 24 & 15 & 12 & 10 & 28 & 8 & 20 & 2.34 \\ \mathrm{R} & \mathrm{LC} & 19 & 17 & 2 & 0 & 9 & 8 & 5 & 6 & 3 & 13 & 9 & 18 & 1.69 \\ \mathrm{~T} & \mathrm{LC} & 0 & 0 & 0 & 0 & 0 & 0 & 1 & 0 & 0 & 0 & 0 & 0 & 0.02\end{array}$

$\mathrm{R}$

LC

0

0

0

1

$\begin{array}{lll}0 & 0 & 0.02\end{array}$

$\mathrm{R}$ 
Tabla 1. Lista de aves registradas en el sitio Ramsar Humedal Lucre-Huacarpay.

Clasificación taxonómica según Plenge (2017). NB = especies que ocurren regularmente en Perú, pero solo en su período no reproductivo, según Plenge (2017). Grado de residencia según Venero (2015): R: Residente, M: Migratoria, T: Temporal, O: Ocasional. Estado de conservación según Decreto Supremo Nº 004-2014-MINAGRI (MINAGRI, 2014): EN: En peligro. VU: Vulnerable. NT. Casi amenazado. LC: Preocupación menor.

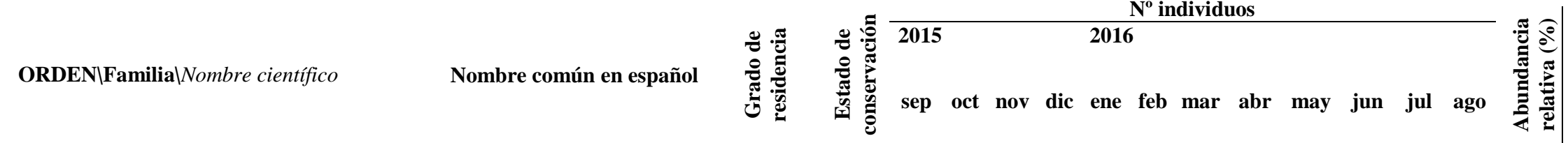

\begin{tabular}{|c|c|c|c|c|c|c|c|c|c|c|c|c|c|c|c|c|}
\hline 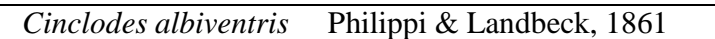 & Churrete de Ala Crema & $\mathrm{R}$ & LC & 1 & 0 & 0 & 0 & 0 & 0 & 0 & 0 & 0 & 1 & 0 & 0 & 0.03 \\
\hline $\begin{array}{l}\text { Phacellodomus striaticeps D'Orbigny \& Lafresnaye, } \\
1838\end{array}$ & Espinero de Frente Rayada & $\mathrm{R}$ & LC & 3 & 3 & 2 & 0 & 0 & 2 & 3 & 0 & 0 & 1 & 0 & 1 & 0.23 \\
\hline Asthenes ottonis Berlepsch, 1901 (E) & Canastero de Frente Rojiza & $\mathrm{R}$ & $\mathrm{LC}$ & 1 & 4 & 3 & 3 & 6 & 2 & 2 & 2 & 4 & 1 & 5 & 5 & 0.59 \\
\hline Tyrannidae & Tiranos atrapamoscas & & & & & & & & & & & & & & & \\
\hline Elaenia albiceps D'Orbigny \& Lafresnaye, 1837 & Fío-Fío de Cresta Blanca & $\mathrm{R}$ & $\mathrm{LC}$ & 0 & 0 & 0 & 0 & 8 & 20 & 17 & 13 & 13 & 5 & 3 & 2 & 1.26 \\
\hline Anairetes flavirostris Sclater \& Salvin, 1876 & Torito de Pico Amarillo & $\mathrm{R}$ & $\mathrm{LC}$ & 0 & 0 & 0 & 0 & 0 & 0 & 2 & 0 & 0 & 0 & 0 & 0 & 0.03 \\
\hline Tachuris rubrigastra Vieillot, 1817 & Siete Colores de la Totora & $\mathrm{R}$ & $\mathrm{LC}$ & 8 & 8 & 6 & 7 & 6 & 4 & 6 & 7 & 5 & 5 & 3 & 3 & 1.06 \\
\hline Lessonia oreas $\quad$ Sclater \& Salvin, 1869 & Negrito Andino & $\mathrm{R}$ & $\mathrm{LC}$ & 1 & 2 & 0 & 0 & 0 & 0 & 0 & 4 & 0 & 1 & 6 & 5 & 0.29 \\
\hline $\begin{array}{l}\text { Muscisaxicola maculirostris d'Orbigny \& Lafresnaye, } \\
1837\end{array}$ & Dormilona Chica & M & $\mathrm{LC}$ & 0 & 0 & 0 & 0 & 0 & 0 & 0 & 1 & 0 & 6 & 2 & 18 & 0.42 \\
\hline $\begin{array}{l}\text { Muscisaxicola rufivertex d'Orbigny \& Lafresnaye, } \\
1837\end{array}$ & Dormilona de Nuca Rojiza & $\mathrm{R}$ & $\mathrm{LC}$ & 8 & 0 & 0 & 0 & 0 & 0 & 1 & 0 & 0 & 4 & 0 & 14 & 0.42 \\
\hline Agriornis montanus d'Orbigny \& Lafresnaye, 1837 & Arriero de Pico Negro & $\mathrm{R}$ & $\mathrm{LC}$ & 1 & 0 & 0 & 0 & 0 & 0 & 0 & 0 & 0 & 1 & 1 & 0 & 0.05 \\
\hline Ochthoeca leucophrys d'Orbigny \& Lafresnaye, 1837 & Pitajo de Ceja Blanca & $\mathrm{R}$ & $\mathrm{LC}$ & 4 & 4 & 1 & 4 & 5 & 1 & 1 & 1 & 0 & 7 & 4 & 0 & 0.50 \\
\hline Tyrannus melancholicus Vieillot, 1819 & Tirano Tropical & $\mathrm{O}$ & $\mathrm{LC}$ & 1 & 0 & 0 & 0 & 0 & 0 & 0 & 0 & 0 & 0 & 0 & 0 & 0.02 \\
\hline Tyrannus tyrannus Linnaeus, 1758 (NB) & Tirano Norteño & $\mathrm{O}$ & $\mathrm{LC}$ & 0 & 0 & 0 & 0 & 0 & 1 & 0 & 0 & 0 & 0 & 0 & 0 & 0.02 \\
\hline Hirundinidae & Golondrinas & & & & & & & & & & & & & & & \\
\hline Orochelidon andecola d'Orbigny \& Lafresnaye, 1837 & Golondrina Andina & $\mathrm{R}$ & $\mathrm{LC}$ & 4 & 8 & 10 & 15 & 0 & 10 & 2 & 2 & 0 & 0 & 0 & 0 & 0.79 \\
\hline Hirundo rustica Linnaeus, 1758 (NB) & Golondrina Tijereta & M & $\mathrm{LC}$ & 6 & 6 & 8 & 0 & 0 & 0 & 0 & 0 & 0 & 0 & 0 & 0 & 0.31 \\
\hline Petrochelidon pyrrhonota Vieillot, 1817 (NB) & Golondrina Risquera & M & $\mathrm{LC}$ & 0 & 0 & 0 & 0 & 1 & 0 & 0 & 0 & 0 & 0 & 0 & 0 & 0.02 \\
\hline Troglodytidae & Ratonas & & & & & & & & & & & & & & & \\
\hline Troglodytes aedon Vieillot, 1809 & Cucarachero Común & $\mathrm{R}$ & $\mathrm{LC}$ & 0 & 0 & 5 & 3 & 2 & 4 & 4 & 2 & 1 & 0 & 0 & 2 & 0.36 \\
\hline Turdidae & Tordos & & & & & & & & & & & & & & & \\
\hline Turdus chiguanco Lafresnaye \& d'Orbigny, 1837 & Zorzal Chiguanco & $\mathrm{R}$ & $\mathrm{LC}$ & 18 & 10 & 10 & 12 & 12 & 12 & 18 & 16 & 18 & 25 & 26 & 27 & 3.17 \\
\hline Thraupidae & Fruteros & & & & & & & & & & & & & & & \\
\hline Pipraeidea bonariensis Gmelin, 1789 & Tangara Azul y Amarilla & $\mathrm{R}$ & $\mathrm{LC}$ & 4 & 1 & 3 & 0 & 7 & 10 & 5 & 4 & 3 & 3 & 6 & 5 & 0.79 \\
\hline Conirostrum cinereum Lafresnaye \& d'Orbigny, 1838 & Pico-de-Cono Cinéreo & $\mathrm{R}$ & $\mathrm{LC}$ & 0 & 3 & 4 & 0 & 2 & 6 & 7 & 3 & 0 & 0 & 0 & 0 & 0.39 \\
\hline Diglossa brunneiventris Lafresnaye, 1846 & $\begin{array}{l}\text { Pincha-Flor de Garganta } \\
\text { Negra }\end{array}$ & $\mathrm{R}$ & LC & 0 & 0 & 0 & 0 & 0 & 0 & 1 & 0 & 0 & 0 & 0 & 0 & 0.02 \\
\hline Diglossa sittoides D'Orbigny \& Lafresnaye, 1838 & Pincha-Flor de Pecho Canela & $\mathrm{O}$ & LC & 0 & 0 & 1 & 0 & 0 & 2 & 0 & 1 & 0 & 0 & 1 & 0 & 0.08 \\
\hline
\end{tabular}


Tabla 1. Lista de aves registradas en el sitio Ramsar Humedal Lucre-Huacarpay.

Clasificación taxonómica según Plenge (2017). NB = especies que ocurren regularmente en Perú, pero solo en su período no reproductivo, según Plenge (2017). Grado de residencia según Venero (2015): R: Residente, M: Migratoria, T: Temporal, O: Ocasional. Estado de conservación según Decreto Supremo Nº 004-2014-MINAGRI (MINAGRI, 2014): EN: En peligro. VU: Vulnerable. NT. Casi amenazado. LC: Preocupación menor.

\begin{tabular}{|c|c|c|c|c|c|c|c|c|c|c|c|c|c|c|c|c|}
\hline \multirow[b]{2}{*}{ ORDENIFamilialNombre científico } & \multirow[b]{2}{*}{ Nombre común en español } & \multirow{2}{*}{ 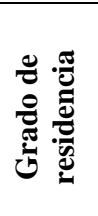 } & \multirow{2}{*}{ 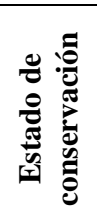 } & \multicolumn{12}{|c|}{$\mathbf{N}^{0}$ individuos } & \multirow{2}{*}{ 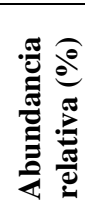 } \\
\hline & & & & sep & oct & nov & dic & ene & feb & mar & abr & may & jun & jul & ago & \\
\hline Phrygilus punensis Ridgway, 1887 & Fringilo Peruano & $\mathrm{R}$ & LC & 0 & 3 & 1 & 1 & 5 & 0 & 1 & 3 & 1 & 1 & 0 & 0 & 0.25 \\
\hline Phrygilus fruticeti Kittlitz, 1833 & Fringilo de Pecho Negro & $\mathrm{R}$ & $\mathrm{LC}$ & 0 & 1 & 0 & 0 & 5 & 3 & 2 & 1 & 1 & 1 & 1 & 3 & 0.28 \\
\hline Phrygilus plebejus Tschudi, 1844 & Fringilo de Pecho Cenizo & $\mathrm{R}$ & $\mathrm{LC}$ & 0 & 1 & 0 & 0 & 0 & 0 & 4 & 2 & 3 & 2 & 2 & 3 & 0.26 \\
\hline Phrygilus alaudinus Kittlitz, 1833 & Fringilo de Cola Bandeada & $\mathrm{R}$ & $\mathrm{LC}$ & 0 & 2 & 0 & 0 & 0 & 0 & 0 & 0 & 0 & 0 & 0 & 3 & 0.08 \\
\hline Sicalis lutea D'Orbigny \& Lafresnaye, 1837 & Chirigüe de la Puna & $\mathrm{T}$ & $\mathrm{LC}$ & 0 & 0 & 0 & 0 & 0 & 4 & 3 & 0 & 0 & 0 & 0 & 0 & 0.11 \\
\hline Sicalis luteola Sparrman, 1789 & Chirigüe Común & $\mathrm{R}$ & $\mathrm{LC}$ & 6 & 0 & 0 & 0 & 0 & 3 & 6 & 0 & 6 & 3 & 1 & 2 & 0.42 \\
\hline Catamenia analis D'Orbigny \& Lafresnaye, 1837 & Semillero de Cola Bandeada & $\mathrm{R}$ & $\mathrm{LC}$ & 44 & 40 & 42 & 28 & 31 & 27 & 39 & 24 & 35 & 35 & 16 & 43 & 6.27 \\
\hline Incertae sedis & Posición incierta & & & & & & & & & & & & & & & \\
\hline Saltator aurantiirostris Vieillot, 1817 & Saltador de Pico Dorado & $\mathrm{R}$ & $\mathrm{LC}$ & 1 & 1 & 3 & 4 & 0 & 6 & 2 & 1 & 0 & 1 & 0 & 1 & 0.31 \\
\hline Emberizidae & Gorriones & & & & & & & & & & & & & & & \\
\hline Zonotrichia capensis Müller, 1776 & Gorrión de Collar Rufo & $\mathrm{R}$ & $\mathrm{LC}$ & 97 & 53 & 45 & 33 & 35 & 40 & 51 & 39 & 57 & 72 & 47 & 97 & 10.33 \\
\hline Icteridae & Ictéridos & & & & & & & & & & & & & & & \\
\hline Agelasticus thilius Molina, 1782 & Tordo de Ala Amarilla & $\mathrm{R}$ & $\mathrm{LC}$ & 37 & 12 & 19 & 20 & 16 & 34 & 46 & 22 & 10 & 14 & 11 & 33 & 4.25 \\
\hline Fringillidae & Pinzones & & & & & & & & & & & & & & & \\
\hline Spinus magellanicus Vieillot, 1805 & Jilguero Encapuchado & $\mathrm{R}$ & $\mathrm{LC}$ & 16 & 20 & 10 & 12 & 20 & 13 & 21 & 14 & 3 & 8 & 9 & 48 & 3.01 \\
\hline
\end{tabular}

${ }^{1}$ Universidad Nacional de San Antonio Abad del Cusco / Perú. BIG BANG CORPORATION EIRL. Autor por correspondencia: wilcard7@ gmail.com.

${ }^{2}$ Universidad Nacional de San Antonio Abad del Cusco / Perú. lizhurtadoc@gmail.com. 